\section{¿Aprender Medicina a distancia?: percepción de estudiantes confinados por la pandemia COVID-19}

\author{
PEDRO BROTONS ${ }^{1,2,3, \#, a}$, MONTSERRAT VIRUMBRALES ${ }^{1, \#, a}$, \\ MARTA ELORDUY ${ }^{1, a}$, PAU MEZQUITA ${ }^{1, a}$, \\ MARIONA GRAELL ${ }^{4, a}$, ALBERT BALAGUER $^{1,5, a}$
}

\section{Perceptions of students confined by the COVID-19 pandemic on distance learning in medicine}

Background: The suspension of face-to-face teaching activity due to the COVID-19 pandemic forced an abrupt transition to distance learning in Spanish universities. Aim: To know how medical students value distance learning in the context of COVID-19 pandemic. Material and Methods: Undergraduate medical students from first to fifth year in Barcelona (Spain) were invited to answer an anonymous online survey about their perceptions and level of satisfaction with virtual learning. Results: Of 483 students invited to the survey, 244 (50.5\%) answered it. Respondents from the first and second year rated distance learning as acceptable (mean 3.1) on a Likert scale from 1 to 5. Those from third to fifth years rated distance learning as unsatisfactory (mean 2.7). The best evaluated aspects were synchronous lectures (3.9) and lectures based on cases (3.4). The worst evaluated issues were motivation (2.3), interaction with faculty (2.1), and additional workload (0.7). Conclusions: The perceptions expressed by these students reinforce the importance of facilitating communication, motivation and participation of students in distance learning in Medicine.

(Rev Med Chile 2020; 148: 1461-1466)

Key words: Education, Distance; Education, Medical, Undergraduate; Personal Satisfaction.

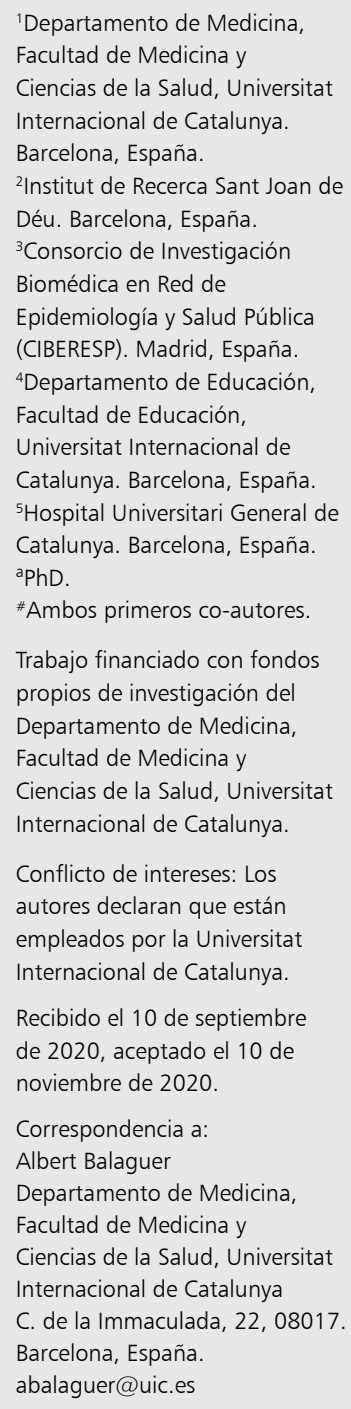

U no de los países más afectados por la emergencia de la enfermedad por el nuevo Coronavirus-19 (COVID-19) ha sido España, con más de 778.000 casos y 31.900 muertes confirmadas hasta el 1 de octubre de $2020^{1}$. El estado de emergencia nacional se declaró el 14 de marzo, imponiendo el estricto confinamiento de la población y la suspensión de la mayoría de las actividades económicas y de toda actividad de ocio, cultural o educativa. Como consecuencia, las universidades españolas se vieron abocadas a una transformación abrupta a la docencia a distancia que ha tenido un impacto considera- ble en Medicina, una disciplina académica en la que la adquisición de habilidades prácticas y las experiencias de aprendizaje colaborativo son fundamentales ${ }^{2-4}$. Para afrontarlo, las Facultades de Medicina procuraron adaptar sus programas y actividades, potenciando un uso más intensivo de los instrumentos de intercomunicación ya existentes. Al mismo tiempo, reforzaron el soporte a profesorado y estudiantes en el manejo de la información y aprendizaje a distancia, así como el acceso y disponibilidad de recursos y material docente en aulas virtuales.

En ese segundo semestre, en la Facultad de 
Medicina de la Universitat Internacional de Catalunya (UIC) (Barcelona, España) se desplegó, precipitadamente, un modelo de docencia virtual basado en la impartición de clases preferentemente sincrónicas, la realización de actividades orientadas al aprendizaje activo (planteamiento y resolución de casos, aprendizaje basado en problemas, foros de consulta y debate) y el fomento del estudio autónomo y autoaprendizaje entre los estudiantes. La aplicación de este modelo virtual se apoyó en un uso extensivo de la plataforma disponible en la Facultad (Moodle Collaborate ${ }^{\mathrm{TM}}$ ). Como resultado del proceso, aplicado con las limitaciones propias de la circunstancia (falta de planificación, profesorado clínico frecuentemente abrumado por situación...), aproximadamente el $60 \%$ de los contenidos previstos para docencia en aula pasaron a ser impartidos en línea, sincrónicamente, y el 40\% de forma asíncrona. Similares modificaciones curriculares han sido introducidas en Facultades de Medicina de otros países ${ }^{5-8}$.

Pero, ¿cómo valoran los estudiantes de Medicina esta transformación digital de la docencia y los diversos instrumentos y metodologías utilizadas? El objetivo del estudio fue describir las percepciones y grado de satisfacción de los estudiantes con la docencia del Grado a distancia.

\section{Métodos}

Estudio transversal descriptivo, efectuado en junio de 2020, de las percepciones de los estudiantes de la Facultad de Medicina de la UIC respecto al cambio a la modalidad de docencia virtual. Esta Facultad admite 100 alumnos por año e imparte el Grado en Medicina desde 2008. La población de estudio fueron los estudiantes de $1^{\circ}$ a $5^{\circ}$ curso. No se incluyeron los estudiantes de $6^{\circ}$, al considerarse distinto el impacto del cambio en dicho curso, dedicado mayoritariamente a la práctica asistencial.

La obtención de datos se efectuó mediante una encuesta anónima en línea utilizando la aplicación Google Forms ${ }^{\mathrm{TM}}$. Las preguntas fueron diseñadas para recoger la percepción de los estudiantes sobre diversos elementos clave de la docencia virtual: motivación, coherencia pedagógica, metodología docente, participación, relación profesor-estudiante, carga de trabajo, aceptación de la formación a distancia y grado de aprendizaje. Estos elementos clave fueron identificados tras revisión de la literatura y validación por parte del equipo investigador. Las respuestas se categorizaron mayoritariamente mediante una escala ordinal de Likert del 1 al 5 (1, nada o malo; 5, mucho o bueno) y se resumieron mediante valores medios y desviaciones estándar.

Se realizaron subanálisis según grupos de cursos, grupos de edad y sexo, comparándose valores medios mediante las pruebas t de Student y ANOVA. Se estableció un valor $\mathrm{p}<0,05$ de significación estadística. Todos los análisis se realizaron con el programa estadístico Stata v.15.1.

La participación en la encuesta fue anónima y los participantes dieron su consentimiento informado previamente. El estudio fue aprobado por el Comité de Ética de la Facultad.

\section{Resultados}

El total de 483 estudiantes matriculados fueron invitados a participar. De ellos, 244 (50,5\%) respondieron a la encuesta, distribuidos entre los cursos $1^{\circ}$ y $2^{\circ}(\mathrm{n}=104,42,6 \%)$ y $3^{\circ}, 4^{\circ}$ y $5^{\circ}$ $(\mathrm{n}=140,57,3 \%)$, predominando las mujeres $(75,6 \%)$ y el rango de edad $18-22$ años $(75,4 \%)$.

Globalmente, sobre una escala de Likert de 1 a 5 , los estudiantes de $1^{\circ}$ y $2^{\circ}$ valoraron el aprendizaje a distancia como aceptablemente satisfactorio (valor medio $\mu=3,1$ ) mientras que los de $3^{\circ}, 4^{\circ} y$ $5^{\circ}$ lo calificaron como poco satisfactorio $(\mu=2,7)$, siendo la diferencia entre ambos grupos significativa $(p<0,01)$. No se observaron diferencias en la valoración del aprendizaje sobre el mismo valor máximo de 5 por grupos de edad (18-20 años: $\mu=3,0$; $21-22$ años: $\mu=2,8$; $23-24$ años: $\mu=2,7$; $>24$ años: $\mu=2,8 ; \mathrm{p}=0,41$ ) ni por sexo (hombres: $\mu=2,7$; mujeres: $\mu=2,9 ; p=0,19)$. En la misma escala, las valoraciones medias de categorías relativas a la docencia a distancia fueron: coherencia pedagógica, expresada por el alineamiento de las actividades virtuales con los objetivos y resultados esperados: $\mu=2,6$; motivación: $\mu=2,3$; relación con los profesores: $\mu=2,1$; carga adicional de trabajo respecto a la acostumbrada presencial: $\mu=0,7$.

Respecto a las metodologías utilizadas, los participantes mostraron sus preferencias por las clases sincrónicas $(\mu=3,9)$ y asincrónicas $(\mu=3,3)$ y su menor interés en las presentaciones básicas $(\mu=2,4)$ y en las presentaciones dinámi- 
cas $(\mu=2,0)$ (Figura 1). El grado de participación fomentado por las diversas tipologías de clases a distancia tuvo valoraciones moderadamente positivas, particularmente las clases basadas en el método del caso $(\mu=3,4)$ (Tabla 1$)$.

El subanálisis de las valoraciones por grupos de cursos reveló percepciones significativamente más favorables de los participantes de los cursos $1^{\circ}-2^{\circ}$ comparados con los de los cursos $3^{\circ}$ a $5^{\circ}$ en valores medios de coherencia pedagógica $(2,7$ vs $2,4, \mathrm{p}=0,02)$, influencia positiva de la formación a distancia en la relación con los profesores $(2,7$ vs $2,4, \mathrm{p}=0,02)$ y carga de trabajo adicional $(0,8$ vs $0,4, p=0,04)$. No se observaron diferencias significativas entre valoraciones de categorías por grupos de edad o sexo.

\section{Discusión}

Las percepciones de los estudiantes de Medicina sobre la docencia a distancia se alinearon en gran medida con las fortalezas y debilidades de esta modalidad identificadas en otros estudios en el ámbito de la educación médica ${ }^{9}$ y en el contexto particular de la pandemia de la COVID-19 ${ }^{10-12}$. En líneas generales, estas percepciones no fueron positivas. La excesiva carga de trabajo (muchos profesores se sintieron obligados a fomentar actividades alternativas extras), la limitada relación con los profesores y una reducida motivación fueron percepciones frecuentemente expresadas por los participantes en la encuesta. Apreciaciones similares han sido descritas en otros estudios sobre el impacto de la COVID-19 en la educación médica universitaria ${ }^{13-15}$. Por otro lado, no se puede obviar el posible impacto de las dificultades familiares y sociales asociadas con la pandemia en el estado de ánimo de los estudiantes y su aceptación del cambio obligado en el modelo educativo ${ }^{16,17}$.

Los estudiantes de los dos primeros cursos manifestaron mejores percepciones que los de los tres cursos siguientes sobre la efectividad del aprendizaje a distancia, la coherencia pedagógica,

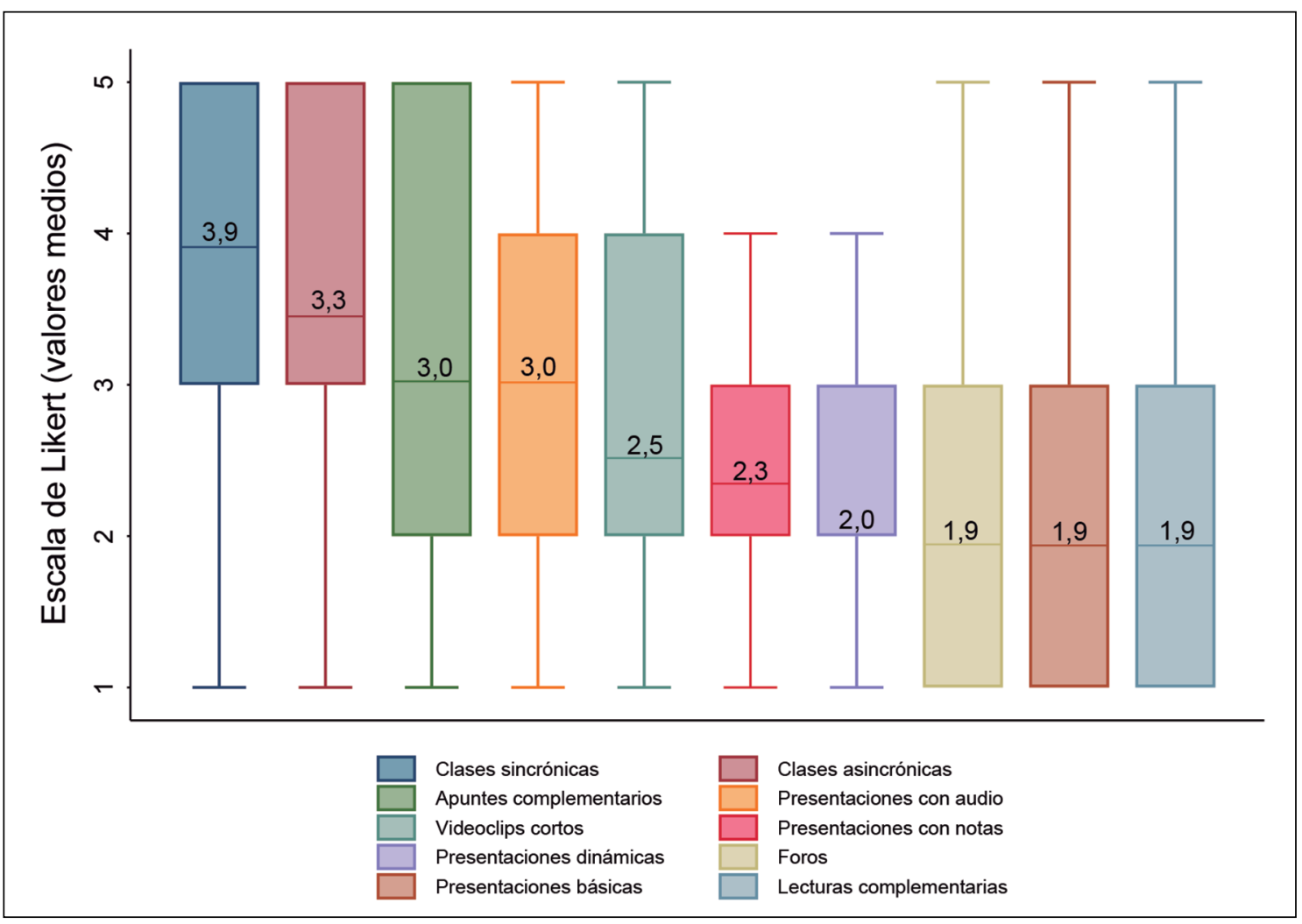

Gráfico 1. Valoración de actividades de docencia a distancia. 
Tabla 1. Categorías, preguntas de la encuesta, opciones de respuesta y valoraciones

\begin{tabular}{|c|c|c|c|c|}
\hline Categorías & Preguntas & Opciones & $\begin{array}{l}\text { Media } \\
\text { (DE)* }\end{array}$ & $\%$ \\
\hline \multirow{6}{*}{$\begin{array}{l}\text { Motivación del } \\
\text { estudiante }\end{array}$} & \multicolumn{2}{|c|}{ ¿La docencia en línea te ha motivado para el aprendizaje? } & $2,3(0,1)$ & \\
\hline & \multirow{5}{*}{$\begin{array}{l}\text { ¿Qué actividades de aprendizaje ha } \\
\text { realizado el profesorado para mantener } \\
\text { la motivación? }\end{array}$} & Aprendizaje lúdico & & $54,9 \%$ \\
\hline & & Presentación de casos & & $54,1 \%$ \\
\hline & & Trabajo/proyecto en grupo & & $52,9 \%$ \\
\hline & & Resolución de problemas & & $37,7 \%$ \\
\hline & & Preguntas reto, otros... & & $<15 \%$ \\
\hline $\begin{array}{l}\text { Coherencia } \\
\text { pedagógica }\end{array}$ & \multicolumn{2}{|c|}{ ¿En qué medida has sabido qué se esperaba de cada sesión? } & $2,6(1,0)$ & \\
\hline \multirow[t]{13}{*}{ Metodología } & \multirow{8}{*}{$\begin{array}{l}\text { ¿Qué actividades realizadas en las } \\
\text { clases destacarías por encima de las } \\
\text { demás? }\end{array}$} & Clases sincrónicas & $3,9(1,3)$ & \\
\hline & & Clases asincrónicas & $3,3(1,7)$ & \\
\hline & & Apuntes complementarios & $3,0(1,7)$ & \\
\hline & & Presentaciones con audio & $3,0(1,5)$ & \\
\hline & & Videoclips cortos complementarios & $2,5(1,6)$ & \\
\hline & & Trabajos/proyectos colaborativos & $2,3(0,1)$ & \\
\hline & & Presentaciones con notas & $2(1,5)$ & \\
\hline & & $\begin{array}{l}\text { Presentaciones, foros, lecturas } \\
\text { complementarias, ... }\end{array}$ & $<2$ & \\
\hline & \multirow{5}{*}{$\begin{array}{l}\text { ¿Qué recomendarías al profesor que } \\
\text { empieza a realizar clases online? }\end{array}$} & Realizar clases magistrales & & $64,3 \%$ \\
\hline & & Realizar actividades dinámicas & & $48,0 \%$ \\
\hline & & Trabajar por píldoras & & $40,2 \%$ \\
\hline & & Generar debates & & $38,9 \%$ \\
\hline & & Proponer talleres, discutir artículos, ... & & $<15 \%$ \\
\hline \multirow[t]{5}{*}{ Participación } & \multirow{5}{*}{$\begin{array}{l}\text { En función de la tipología de clases } \\
\text { online, valora el grado de participación }\end{array}$} & Método de caso & $3,4(1,2)$ & \\
\hline & & Clases teórico-prácticas & $3,1(1,3)$ & \\
\hline & & Tutorías de proyectos en equipo & $3,1(1,4)$ & \\
\hline & & Clase magistral & $2,9(1,3)$ & \\
\hline & & Laboratorio & $2,7(1,5)$ & \\
\hline \multirow{4}{*}{$\begin{array}{l}\text { Relación } \\
\text { profesor- } \\
\text { estudiante }\end{array}$} & \multicolumn{2}{|c|}{$\begin{array}{l}\text { La situación online provocada por la COVID-19, ¿ha influido positivamente en la } \\
\text { relación con el profesor/a? }\end{array}$} & $2,1(1,1)$ & \\
\hline & \multirow{3}{*}{$\begin{array}{l}\text { ¿Qué recursos se han empleado con } \\
\text { mayor asiduidad para comunicarte con } \\
\text { el profesor/a? }\end{array}$} & A través de los delegados & & $78,3 \%$ \\
\hline & & Mails & & $76,2 \%$ \\
\hline & & Foros & & $24,2 \%$ \\
\hline $\begin{array}{l}\text { Carga de } \\
\text { trabajo }\end{array}$ & \multicolumn{2}{|c|}{ Valora la carga de trabajo de la docencia online respecto a la presencial } & $0,7(0,7)$ & \\
\hline \multirow{5}{*}{$\begin{array}{l}\text { Aceptación de } \\
\text { la formación a } \\
\text { distancia }\end{array}$} & \multirow[t]{2}{*}{ ¿Te gusta aprender de forma online? } & No & & $69,3 \%$ \\
\hline & & Sí & & $30,7 \%$ \\
\hline & \multirow{3}{*}{$\begin{array}{l}\text { ¿Has aprendido más o menos en clases } \\
\text { online, respecto a la presencial? }\end{array}$} & Menos & & $63,1 \%$ \\
\hline & & Igual & & $29,1 \%$ \\
\hline & & Más & & $7,8 \%$ \\
\hline Aprendizaje & \multicolumn{2}{|l|}{ ¿Cómo valoras el aprendizaje online? } & $2,9(1,2)$ & \\
\hline
\end{tabular}

*Medias y desviaciones estándar (DE), sobre una escala Likert de 1 a 5; siendo $1=$ nada (mal) y 5 = máximo (bien). 
la relación con el profesorado y la carga de trabajo asociada. Estas diferencias podrían relacionarse con perfiles diferenciados de estudiantes y profesores en ambos tramos del Grado, así como con el distinto peso relativo de los contenidos teóricos y prácticos en cada tramo. Es probable que las expectativas de los estudiantes de primeros cursos respecto a la docencia a distancia fueran menores, así como menor su preocupación e implicación en labores de soporte asistencial y voluntariado en relación con la pandemia. Las diferentes características del profesorado también pudieron ser determinantes. En los primeros cursos en el centro de estudio se cuenta con mayor número de profesores con dedicación exclusiva o muy preferente a la docencia y a la investigación mientras que en los últimos cursos el profesorado está principalmente dedicado a la práctica médica y quedó en gran medida expuesto a alta presión asistencial durante la pandemia. Este factor diferencial pudo influir en una mayor disponibilidad de los docentes de los primeros cursos para capacitarse rápidamente en el desarrollo de contenidos digitales, en comparación con los de los últimos cursos. Asimismo, pudo favorecer una mayor coherencia pedagógica de las actividades, una interacción más estrecha con los estudiantes y una mejor coordinación de cargas de trabajo en los primeros cursos. Finalmente, se podría especular que la propia naturaleza de los contenidos, más teóricos y menos aplicados en las asignaturas de los primeros cursos, puede haber facilitado la traslación de dichos contenidos teóricos a entornos virtuales, suscitando una mayor aceptabilidad de la docencia a distancia entre los estudiantes en el primer tramo del Grado.

Entre las limitaciones del estudio, debe reconocerse que, aunque el nivel de respuesta de la encuesta fue aceptable (aproximadamente la mitad de los estudiantes matriculados en el Grado) no es descartable cierto sesgo de participación. Las particularidades de la propia Facultad tampoco permiten una extrapolación directa a otros entornos universitarios.

Sin embargo, los resultados obtenidos apuntan claves interesantes para mejorar la docencia a distancia de Medicina en situaciones que pueden repetirse en el futuro. La importancia de mantener horarios y sesiones sincrónicas (mejor con contenidos que queden disponibles grabados), la potenciación de los métodos del caso y aque- llas actividades que fomenten la participación activa y la motivación de los estudiantes, son elementos clave a potenciar. De la misma forma, debería favorecerse una adecuada planificación y coordinación de las cargas de trabajo y la mejor comunicación fluida entre profesores, estudiantes y dirección del Grado, que debería extremar su apoyo al profesorado clínico.

En conclusión, los estudiantes de Medicina manifestaron una percepción crítica de la docencia a distancia, que no se puede disociar de limitaciones intrínsecas de este modelo para promover el aprendizaje colaborativo ni de su aplicación en el contexto de emergencia educativa de la pandemia. Las precepciones observadas refuerzan la importancia de facilitar la comunicación, motivación y participación activa de los estudiantes de Medicina en el proceso de aprendizaje a distancia.

Agradecimientos: Agradecemos la colaboración de los estudiantes de Grado de la Facultad de Medicina de la Universitat Internacional de Catalunya su participación en la encuesta que ha facilitado la realización de este estudio.

\section{Referencias}

1. CCAES Centro de Coordinación de Alertas y Emergencias Sanitarias. Actualización no 219. Enfermedad por coronavirus (COVID-19). 01.10.2020. https:/www. mscbs.gob.es/en/profesionales/saludPublica/ccayes/ alertasActual/nCov-China/documentos/Actualizacion_219_COVID-19.pdf [Consultada 20/10/2020] .

2. Ferrel MN, Ryan JJ. The impact of COVID-19 on medical education. Cureus. 2020;12: e7492.

3. Rolak S, Keefe AM, Davidson EL, Aryal P, Parauji S. Impacts and challenges of United States medical students during the COVID-19 pandemic. World J Clin Cases 2020; 8 (15): 3136-41.

4. Rose S. Medical student education in the time of COVID-19. JAMA.2020;10.1001/jama.2020.5227.

5. Bączek M, Zagańczyk-Bączek M, Szpringer M, Jaroszynski A, Wozakowska-Kaplon B. Students' perception of online learning during the COVID-19 pandemic: a survey study of Polish medical students. 2020. doi: 10.21203/rs.3.rs-41178/v1.

6. Khalil R, Mansour AE, Fadda WA, Almisnid K, Aldamegh M, Al-Nafeesah A, et al. The sudden transition to synchronized online learning during the COVID-19 pandemic in Saudi Arabia: a qualitative study exploring 
medical students' perspectives. BMC Med Educ 2020; 20 (1): 285 .

7. Sandhaus Y, Kushnir T, Ashkenazi S. Electronic Distance Learning of Pre-clinical Studies During the COVID-19 Pandemic: A Preliminary Study of Medical Student Responses and Potential Future Impact. Isr Med Assoc J 2020; 8 (22): 423-7.

8. Shahrvini BB, Baxter, Coffey CS, MacDonald BBV, Lander SL. Pre-Clinical Remote Undergraduate Medical Education During the COVID-19 Pandemic: A Survey Study. Preprint. Res Sq 2020; rs.3.rs-33870. doi: 10.21203/rs.3.rs-33870/v1.

9. O'Doherty D, Dromey M, Lougheed J, Hannigan A, Last J, McGrath D. Barriers and solutions to online learning in medical education - an integrative review. BMC Med Educ 2018; 18 (1): 130.

10. Hodges C, Moore S, Lockee B, Trust T, Bond A. EDUCAUSE Review. The difference between emergency remote teaching and online learning. Available at: https://er.educause.edu/articles/2020/3/the-difference-between-emergency-remote-teaching-and-online-learning [Accessed 3/9/2020].

11. Emmanuel EJ. The inevitable Reimagining of Medical Education. JAMA 2020; 323 (12): 1127-8.

12. Cleland J, McKimm J, Fuller R, et al. Adapting to the impact of COVID-19: Sharing stories, sharing practice. Med Teach 2020; 42 (7): 772-5.

13. Khalil R, Mansour AE, Fadda WA, Almisnid K, Aldamegh M, Al-Nafeesah A, et al. The sudden transition to synchronized online learning during the COVID-19 pandemic in Saudi Arabia: a qualitative study exploring medical students' perspectives. BMC Med Educ 2020; 20 (1): 285.

14. Bączek M, Zagańczyk-BączekM, Szpringer M, Jaroszynski A, Wozakowska-Kaplan B. Students' perception of online learning during the COVID-19 pandemic: a survey study of Polish medical students. 2020. doi: 10.21203/rs.3.rs-41178/v1.

15. Shahrvini BB, Baxter, Coffey CS, MacDonald BBV, Lander SL. Pre-Clinical Remote Undergraduate Medical Education During the COVID-19 Pandemic: A Survey Study. Preprint. Res Sq 2020; rs.3.rs-33870. doi: 10.21203/rs.3.rs-33870/v1

16. Ullah R, Amin S. The psychological impact of COVID-19 on medical students. Psychiatry Res 2020; 288: 113020.

17. Meo SA, Abukhalaf AA, Alomar AA, Sattar K, Klonoff DC. COVID-19 Pandemic: Impact of Quarantine on Medical Students' Mental Wellbeing and Learning Behaviors. Pak J Med Sci 2020; 36 (COVID19-S4): S43-8. 